\title{
Macro EMG in healthy subjects of different ages
}

\author{
ERIK STÅLBERG, PETER RW FAWCETT* \\ From the Department of Clinical Neurophysiology, University Hospital, Uppsala, Sweden
}

SUMMARY The findings of the recently developed technique of Macro EMG in healthy subjects of different ages are described. The characteristics of the Macro motor unit potential which probably reflects the relative size of the whole motor unit are presented together with suggested mormal data for the three muscles studied. An increase in size of the Macro motor unit potential was found with age, particularly after the age of 60 years. Possible factors determining the Macro EMG signal and the age related changes are discussed.

The spike component of the so called motor unit potential (MUP) recorded in conventional EMG with a concentric needle electrode is generated by only a small proportion of all the muscle fibres in a motor unit. ${ }^{1}$ Action potentials from more fibres, but not all, contribute to the initial and terminal phases, from which the duration is determined. No information is obtained about the size of the motor unit comprising the total number and size of component fibres with this technique. Knowledge of this parameter would be of great value in the understanding of normal physiological and pathophysiological mechanisms in muscle and in particular may help to differentiate between degenerative and regenerative processes which lead to alterations of motor unit size. Macro EMG is a recently developed technique ${ }^{2}$ which seems to provide this information. This report deals with findings in muscles of healthy subjects of different ages and forms the basis for the further use of the method in patients with neuromuscular disorders.

\section{Methods}

The recording electrode consists of a modified SFEMG electrode with a platinum wire $25 \mu \mathrm{m}$ in diameter exposed in a side-port $7.5 \mathrm{~mm}$ behind the tip. The steel cannula $0.55 \mathrm{~mm}$ in diameter is insulated to within $15 \mathrm{~mm}$ of the tip. Recordings are made on two channels. On one the derivation is made between the electrode shaft and the central core of a subcutaneously inserted concentric needle

${ }^{*}$ Present address: Muscular Dystrophy Group Research Laboratories, Newcastle General Hospital, Westgate Road, Newcastle upon Tyne, England.

Address for reprint requests: Dr E Stälberg, University Hospital, Dept of Clinical Neurophysiology, S-751 85, Uppsala 14, Sweden.

Received 2 April 1982. Accepted 28 June 1982 electrode placed remotely from the muscle under investigation. Filter settings are $8 \mathrm{~Hz}-8 \mathrm{kHz}$. On the other channel a SFEMG derivation is made between the small surface and the electrode shaft. Filter settings are $500 \mathrm{~Hz}-16 \mathrm{kHz}$. The SFEMG recording is used to trigger the oscilloscope. The signal from the electrode shaft is delayed usually about $40 \mathrm{~ms}$ and then averaged by which means the action potential of the synchronously firing motor unit is extracted, the Macro motor unit potential (Macro MUP). The averaging procedure has been performed in two different ways: (a) initially by using an external averager included in the EMG equipment, (b) later by using a microprocessor. In the latter technique A/D conversion is started at the time of the SFEMG action potential. The delayed signal is alternatively averaged in one of two buffer stores. By point-topoint subtraction of the signals in the two stores and adding the absolute values of these differences a value is obtained indicating the dissimilarity between the averaged signals. This provides a "quality control" of the averaging process. ${ }^{3}$ When the difference falls below a preset value the average of the two signals is obtained for final analysis. In order to ensure that activity from the same motor unit is studied throughout each acquisition the triggering SFEMG recording is continously checked at a higher sweep speed, usually $0.5 \mathrm{~ms} / \mathrm{div}$ on a separate oscilloscope. The number of single fibre components in the triggering action potential with an amplitude exceeding $200 \mu \mathrm{V}$ and duration of less than 300 $\mu \mathrm{s}$ is counted. The mean value of this number after recording from 20 different sites is calculated and called fibre density. ${ }^{4}$ While searching for a new recording site only the single fibre EMG recording is monitored in order to avoid bias in the selection of motor units. The amplitude of the SFEMG potentials does not reflect that of the Macro MUP. Each Macro MUP is displayed on a TV monitor after the averaging process is completed and also later as a print out as the end of the study (fig 1). When two or more Macro MUP recordings appear to be very similar in shape and size, the second and subsequent recordings are discarded. For each investigation the aim is to obtain at least 20 different Macro MUPs. These are recorded at different depths of the muscle using two to five different skin insertions. A complete investigation requires about 20 minutes. 

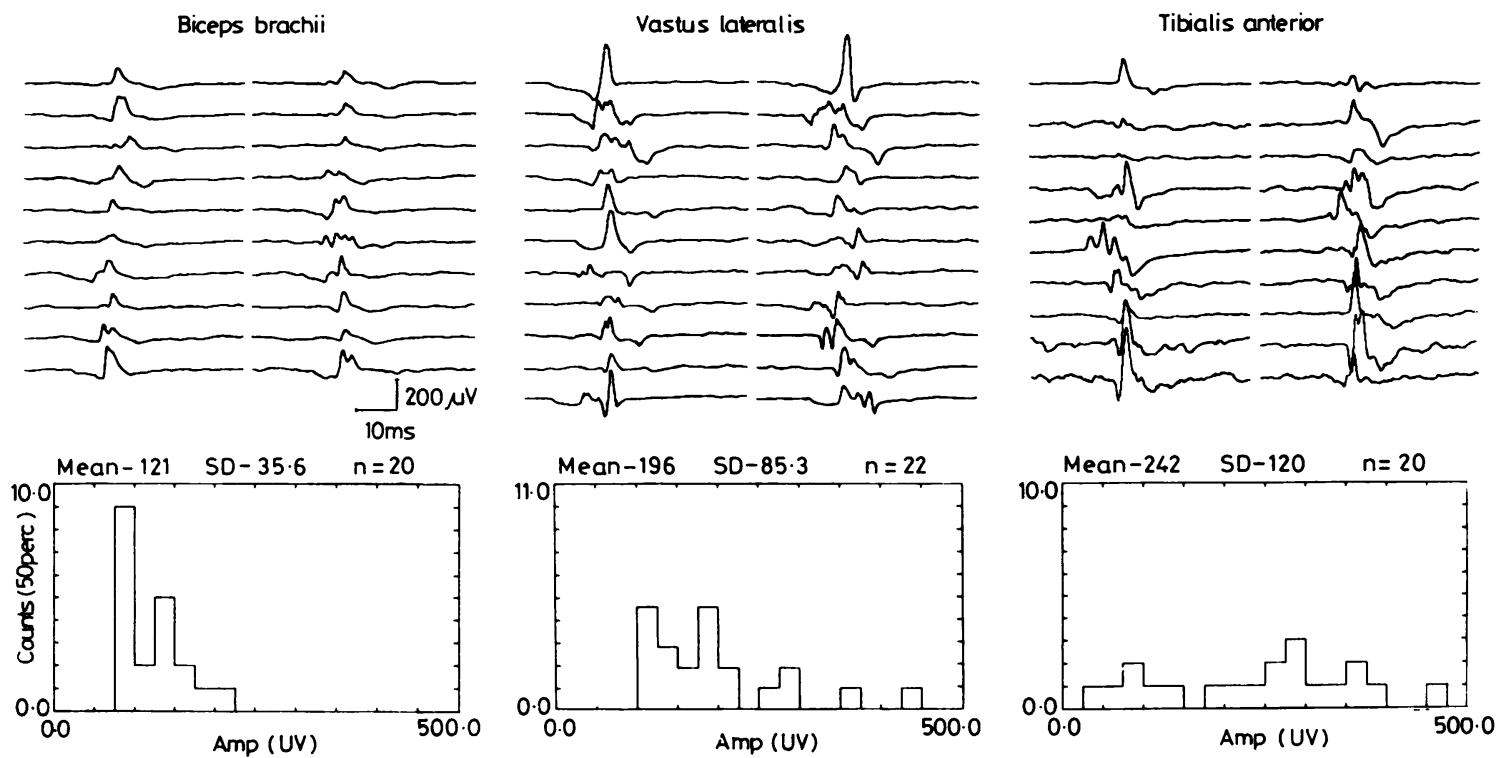

Fig 1 Macro EMG recordings from three muscles in the same healthy subject, aged 37. The picture shows individual Macro MUPs and histograms of their amplitudes. Note slightly skewed distribution of the values in the vastus lateralis muscle.

All recordings are made with slight muscle activation which is typically less than $30 \%$ of maximal force. This implies that the recordings are mostly from low threshold motor units.

\section{Analysis}

A period of $80 \mathrm{~ms}$ is A/D converted with the signal delayed about $40 \mathrm{~ms}$ relative to the triggering single fibre action potential in order to see the earlier appearing components. The first and last $5 \mathrm{~ms}$ are omitted to avoid artefacts. Of the remaining signal the mean value of the first and last 5 $\mathrm{ms}$ is used to establish a baseline for correction of the signal from the 10 th to the 70 th ms. The Macro MUP is analysed with regard to its maximal peak-to-peak amplitude (fig 1) and the area under the signal during the epoch of 60 ms by means of the computer. It should be noted that the amplitude measurements can also be made manually from film recordings. In the present study a manual measurement of the number of peaks and time between the extreme peaks is made. Other, and perhaps more efficient approaches to describe the inherent characteristics of the Macro MUP are being examined at the moment, one of which is a signal definition based on functions derived from Hermite polynomials. ${ }^{5}$ Since these methods all require computer facilities and since the amplitude seems to be a very important descriptor, we will restrict analysis in the present study to this and the previously mentioned parameters.

\section{Statistics}

From the data in each subject the following amplitude parameters were sought for each decade; the mean value and the mean and SD of the median values from each subject. The range of individual Macro MUP amplitudes and the range of median values were noted. The mean and $\mathrm{SD}$ of the median area values was also calculated (table 1).

Defunition of normality From the obtained data "normal values" will be defined for the use in Macro EMG studies in patients. The normal values should be easy to use, meaningful, and define the normal material as closely as possible on the one hand to avoid any "healthy subject" being classified as abnormal when using the data, and on the other hand to provide limits for normality which are as narrow as possible. The mean values in the individual cases are not very useful because of the skewed distribution of the Macro MUP amplitudes which occurred particularly at higher ages. Therefore median values are obtained, and their mean has been determined for each decade. To assess lower and upper limits for individual Macro MUPs the following procedure was used. The extreme values at each end of the amplitude range were discarded in each subject, providing lower and upper limits beyond which only one potential of whatever amplitude may be accepted as being normal (fig 2). The limit for each decade was then set at the remaining minimum and maximum potential amplitude when all the subjects within that decade were taken into account. This form of treatment is applied to obtain a better definition of the lower and upper limits of the majority of data without it being disturbed by occasional extreme values. As a consequence of too small material to date these limit values differ, sometimes quite considerably, between successive decades. Therefore the suggested limits are made somewhat wider for some decades to fit the values of the preceding and following decades. In no decade 
Table 1 Macro EMG results

\begin{tabular}{|c|c|c|c|c|c|c|c|c|c|c|c|c|}
\hline \multirow[t]{2}{*}{ Age yr } & \multicolumn{4}{|c|}{ Biceps } & \multicolumn{4}{|c|}{ Vastus Lateralis } & \multicolumn{4}{|c|}{ Tibialis anterior } \\
\hline & No & $\begin{array}{l}\text { Median * } \\
\text { Amp }\end{array}$ & $S D$ & $\begin{array}{l}\text { Median }{ }^{\dagger} \\
\text { Area }\end{array}$ & No & $\begin{array}{l}\text { Median* } \\
\text { Amp }\end{array}$ & $S D$ & $\begin{array}{l}\text { Median } \dagger \\
\text { Area }\end{array}$ & No & $\begin{array}{l}\text { Median* } \\
\text { Amp }\end{array}$ & $S D$ & $\begin{array}{l}\text { Median } \dagger \\
\text { Area }\end{array}$ \\
\hline $\begin{array}{l}10-19 \\
20-29 \\
30-39 \\
40-49 \\
50-59 \\
60-69 \\
70-79\end{array}$ & $\begin{array}{r}3 \\
6 \\
11 \\
7 \\
8 \\
6 \\
2\end{array}$ & $\begin{array}{r}69 \\
100 \\
99 \\
115 \\
110 \\
122 \\
98\end{array}$ & $\begin{array}{r}3.6 \\
22.2 \\
45.7 \\
30.6 \\
30.9 \\
63.2 \\
3.5\end{array}$ & $\begin{array}{l}417 \\
627 \\
693 \\
645 \\
575 \\
699 \\
638\end{array}$ & $\begin{array}{r}4 \\
20 \\
22 \\
25 \\
24 \\
18 \\
26\end{array}$ & $\begin{array}{l}112 \cdot 3 \\
163 \cdot 3 \\
156 \cdot 3 \\
150 \cdot 9 \\
165 \cdot 5 \\
202 \cdot 5 \\
208 \cdot 3\end{array}$ & $\begin{array}{l}11 \cdot 5 \\
43 \cdot 0 \\
44 \cdot 0 \\
46 \cdot 6 \\
70 \cdot 3 \\
102 \cdot 8 \\
114\end{array}$ & $\begin{array}{l}490 \\
760 \cdot 5 \\
757 \cdot 5 \\
720 \cdot 9 \\
789 \cdot 8 \\
948 \cdot 0 \\
970 \cdot 8\end{array}$ & $\begin{array}{l}4 \\
8 \\
9 \\
7 \\
7 \\
5 \\
6\end{array}$ & $\begin{array}{l}132 \\
158 \\
159 \\
207 \\
190 \\
207 \\
351\end{array}$ & $\begin{array}{c}39 \cdot 2 \\
46 \cdot 7 \\
49 \cdot 5 \\
95 \cdot 7 \\
98 \cdot 2 \\
63 \cdot 7 \\
170\end{array}$ & $\begin{array}{r}700 \\
875 \\
854 \\
1023 \\
920 \\
1033 \\
1579\end{array}$ \\
\hline
\end{tabular}

* Mean and SD value of the individual median amplitude values from each subject for each decade.

t Mean value of the individual median area values from each subject for each decade.

No: indicates number of subjects studied in each decade.

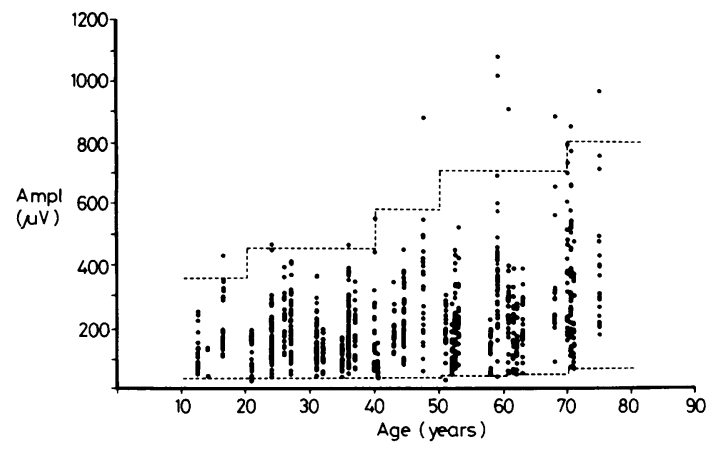

Fig 2 Plot of amplitudes of individual Macro MUPs in tibialis anterior muscles of healthy subjects. Suggested normal limits (....), set after discarding one extreme value in each subject.

are the limits narrower than they were originally. The given values were in fact close to $2 \mathrm{SD}$ from the mean value of the individual median values in most of the decades.

\section{Results}

In three muscles four thousand seven hundred and nine recordings were made; biceps brachii $(43$ muscles), vastus lateralis (135 muscles) and tibialis anterior (46 muscles) in subjects aged between 12 and 75 years, 107 from women and 117 from men, without symptoms or signs of earlier or present neuromuscular disorders. The results are summarised in fig 3 and table 1.

\section{AMPLITUDE}

As can be seen in fig 2 there is a great scatter in the motor unit amplitudes with a range of between four to 10 times in the individual subjects under the age of 60 , and up to 30 times at higher ages. The maximal value ever seen in subjects below 60 years of age was $595 \mu \mathrm{V}$ for biceps, $1068 \mu \mathrm{V}$ for vastus lateralis and $1077 \mu \mathrm{V}$ for tibialis anterior muscles, while above 60 years of age the values were $704 \mu \mathrm{V}$, $1611 \mu \mathrm{V}$ and $962 \mu \mathrm{V}$ respectively. The distribution of amplitudes is not always Gaussian usually showing a slight positive skewness, particularly in the elderly. The mean value is therefore usually higher than the median. Within each decade there is a variation of the individual mean values which, particularly in vastus lateralis and tibialis anterior differ significantly in many decades. For example in the fifth decade in tibialis anterior muscle the extreme mean values ranged from $64 \pm 24.5$ (median 64$)$ to $330 \pm 214$ (median 328) $\mu \mathrm{V}(\mathrm{p}<0.001)$. Regression analysis of individual data and of median values against age shows that in the case of biceps there is no significant change. In contrast in tibialis anterior there is a slight but significant increase up to the age of 60 and thereafter in both vastus lateralis and tibialis anterior there is a quite marked increase in amplitude. The median values in both muscles for ages above 60 are significantly higher than for the ages below $(\mathrm{p}<0.001)$.

\section{A REA}

For each muscle there is a close correlation between amplitude and area of the recorded signal (fig 4A). The amplitude/area ratio is similar for the different muscles, the average values for each muscle ranging from 3.4 to 3.7 without any systematic differences between decades. A greater scatter of the ratio was noted for individual subjects, ranging from a minimum value of 1.6 to a maximum value of 6.4 . If in any of the recorded Macro MUPs this ratio exceeds $3 \mathrm{SD}$ of the material of 20 data in each subject it usually indicates an artefact such as a transient, noise or abnormal potential shape. Because of this close correlation between amplitude and area we have not found it necessary to report all the area data. The median area values are presented for comparison with the amplitude data (table 1). 

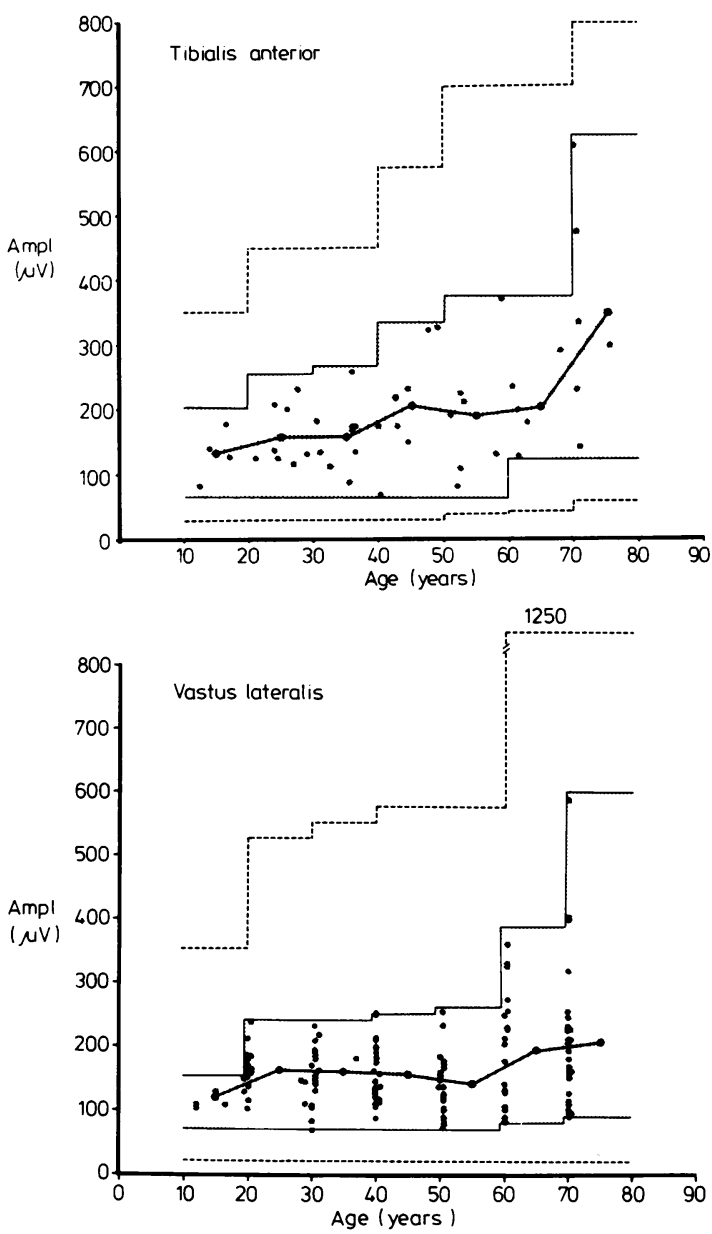

NORMAL VALUES

The normal values calculated as described under Methods are summarised in fig 3 and table 2. Using the given criteria a recording from a case is "abnormal" either if the median value is outside the given limits, or if more than one Macro MUP is outside the given limits for the individual potentials. An abnormality may however also be detected in the case of an abnormal distribution of values within these limits, particularly in patients with predominantly small Macro MUPs. This could for example be analysed by plotting cumulative distributions of the data.

SEX

Too few subjects were studied to allow proper statistical analysis of the difference between the sexes in each decade, and this factor has therefore been assessed for the pooled data in the age ranges 15-

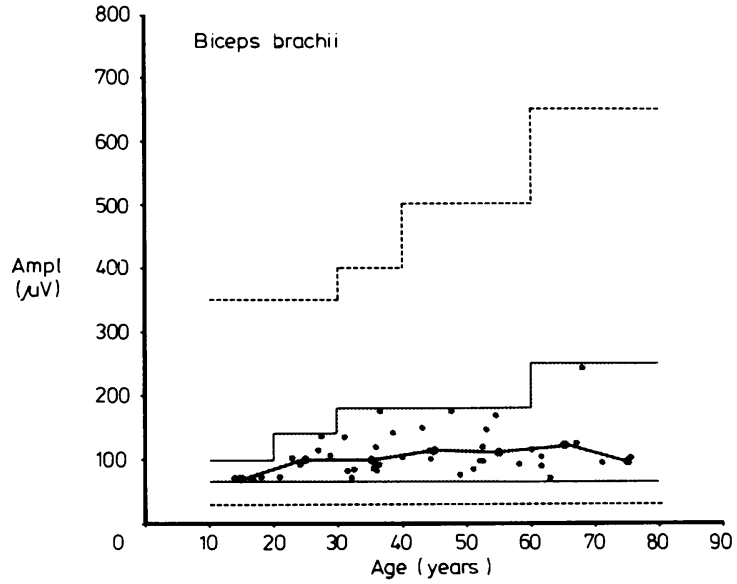

Fig 3 Plot of the median values from each subject and their mean for each decade from biceps brachii, vastus lateralis and tibialis muscles. Shaded area defines the suggested normal limits for the median values. Hatched line defines the suggested normal limits for individual Macro MUP amplitudes.

$39,40-59$ and 60-75. For each age range in each muscle, the means of the pooled individual Macro MUP values of the women were slightly but significantly smaller than those of the men, $(p<$ 0.001-0.05). However when the mean of the median values for each age range were compared, although the trend remained, the difference was statistically significant for only the 40-59 age group in biceps (fig 4).

\section{MUP SHAPE}

The shape of the Macro MUP showed slight differences among the three muscles studied (fig 1, table 3 ). In biceps, potentials tended to have a relatively simple configuration with mainly single and double peaks. In tibialis anterior potentials with two or more peaks occurred more frequently, and in vastus lateralis complex potentials were common. The range of time intervals between the extreme peaks 

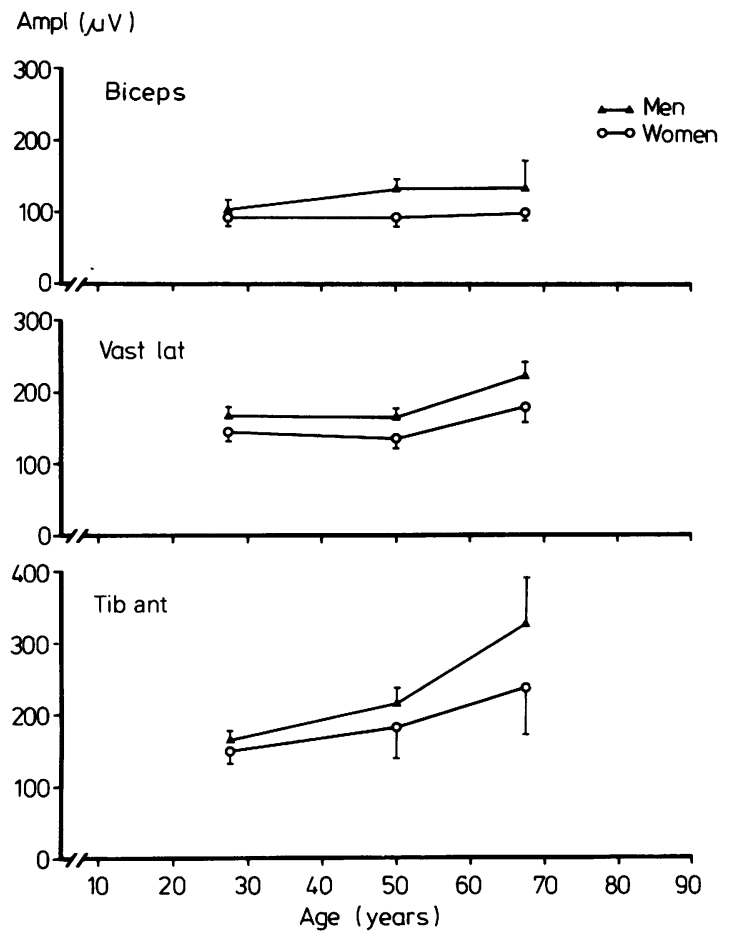

Fig 4 The mean of the median amplitude values for males $(\Delta)$ and females $(O)$ in three age groups. The median values are significantly different $(p<0.05)$ only for biceps, age range 40 to 59. Bars indicate $S E$ of the mean of median values. in each Macro MUP was similar in each muscle with no significant difference between those from subjects below and those above the age of 60 . The interpeak interval (MIPeakI) was about $3 \mathrm{~ms}$ in all three muscles and was uninfluenced by age.

\section{AMPI_ITUDE-FD}

There was no correlation between the number of spikes in the triggering potential and the same motor unit Macro MUP. However the fibre density showed a tendency to increase after the age of 50 (tibialis anterior) or 60 (biceps and vastus lateralis), and there was a slight but significant correlation between median amplitude and fibre density $(r=0 \cdot 2-0 \cdot 3 ; p$ $<0.05)$ and median area and fibre density $(\mathrm{r}=$ $0 \cdot 2-0 \cdot 3 ; p<0 \cdot 05)$ in all three muscles.

\section{AMPLITUDE-DEPTH}

In each of two subjects motor units were recorded at measured depths from skin to bone in biceps or vastus lateralis muscles. In the first case there was no significant change in amplitude among 41 motor unit potentials. In the other there was a tendency for increasing amplitude with depth among 21 recordings from vastus lateralis $(\mathrm{r}=0.44, \mathrm{p}<0.05)$ corresponding to a doubling in amplitude when the electrode was moved $45 \mathrm{~mm}$ deeper in the muscle.

\section{AMPLITUDE-POSITION}

The effect of position of the recording electrode within the same motor unit was studied in the biceps brachii muscle in five subjects. A separate SFEMG

Table 2 Suggested amplitude limits

\begin{tabular}{|c|c|c|c|c|c|c|c|c|c|c|c|c|}
\hline \multirow[t]{3}{*}{ Age yr } & \multicolumn{4}{|c|}{ Biceps } & \multicolumn{4}{|c|}{ Vastus Lateralis } & \multicolumn{4}{|c|}{ Tibialis anterior } \\
\hline & \multicolumn{2}{|c|}{ Median* } & \multicolumn{2}{|c|}{$\begin{array}{l}\text { Individual } \dagger \\
\text { Macro MUP }\end{array}$} & \multicolumn{2}{|c|}{ Median* } & \multicolumn{2}{|c|}{$\begin{array}{l}\text { Individual }{ }^{\dagger} \\
\text { Macro MUP }\end{array}$} & \multicolumn{2}{|c|}{ Median* } & \multicolumn{2}{|c|}{$\begin{array}{l}\text { Individual } \dagger \\
\text { Macro MUP }\end{array}$} \\
\hline & $\min$ & $\max$ & $\min$ & $\max$ & $\min$ & $\max$ & $\min$ & $\max$ & $\min$ & $\max$ & $\min$ & $\max$ \\
\hline $\begin{array}{l}10-19 \\
20-29 \\
30-39 \\
40-49 \\
50-59 \\
60-69 \\
70-79\end{array}$ & $\begin{array}{l}65 \\
65 \\
65 \\
65 \\
65 \\
65 \\
65\end{array}$ & $\begin{array}{l}100 \\
140 \\
180 \\
180 \\
180 \\
250 \\
250\end{array}$ & $\begin{array}{l}30 \\
30 \\
30 \\
30 \\
30 \\
30 \\
30\end{array}$ & $\begin{array}{l}350 \\
350 \\
400 \\
500 \\
500 \\
650 \\
650\end{array}$ & $\begin{array}{l}70 \\
70 \\
70 \\
70 \\
70 \\
80 \\
90\end{array}$ & $\begin{array}{l}150 \\
240 \\
240 \\
250 \\
260 \\
370 \\
600\end{array}$ & $\begin{array}{l}20 \\
20 \\
20 \\
20 \\
20 \\
20 \\
20\end{array}$ & $\begin{array}{r}350 \\
525 \\
550 \\
575 \\
575 \\
1250 \\
1250\end{array}$ & $\begin{array}{r}65 \\
65 \\
65 \\
65 \\
65 \\
120 \\
120\end{array}$ & $\begin{array}{l}200 \\
250 \\
260 \\
330 \\
375 \\
375 \\
620\end{array}$ & $\begin{array}{l}30 \\
30 \\
30 \\
30 \\
40 \\
45 \\
65\end{array}$ & $\begin{array}{l}350 \\
450 \\
450 \\
575 \\
700 \\
700 \\
800\end{array}$ \\
\hline
\end{tabular}

*Suggested minimum and maximum limits for the median amplitude value for any one subject in each decade.

† Suggested minimum and maximum limits for the individual Macro MUP amplitudes for any one subject in each decade after discarding the extreme values at each end of the amplitude range.

Table 3 Macro MUP configuration

\begin{tabular}{|c|c|c|c|c|c|c|}
\hline & \multicolumn{4}{|c|}{ \% Incidence of peaks } & \multirow{2}{*}{$\begin{array}{l}\text { Interval between extreme peaks } \\
\text { Range ms }\end{array}$} & \multirow{2}{*}{$\frac{\text { Mean inter-peak interval }}{(\text { MI Peak I) ms }}$} \\
\hline & One & Two & Three & Four & & \\
\hline $\begin{array}{l}\text { Biceps } \\
\text { Tib. Ant. } \\
\text { Vast. Lat. }\end{array}$ & $\begin{array}{l}55 \\
33 \\
27\end{array}$ & $\begin{array}{l}36 \\
41 \\
39\end{array}$ & $\begin{array}{r}7 \\
22 \\
22\end{array}$ & $\begin{array}{r}2 \\
4 \\
12\end{array}$ & $\begin{array}{l}0 \cdot 3-14 \cdot 3 \\
1 \cdot 6-12 \cdot 0 \\
0 \cdot 8-12 \cdot 8\end{array}$ & $\begin{array}{l}3.1 \pm 1.6 \\
3.4 \pm 1.5 \\
3.2 \pm 1.5\end{array}$ \\
\hline
\end{tabular}




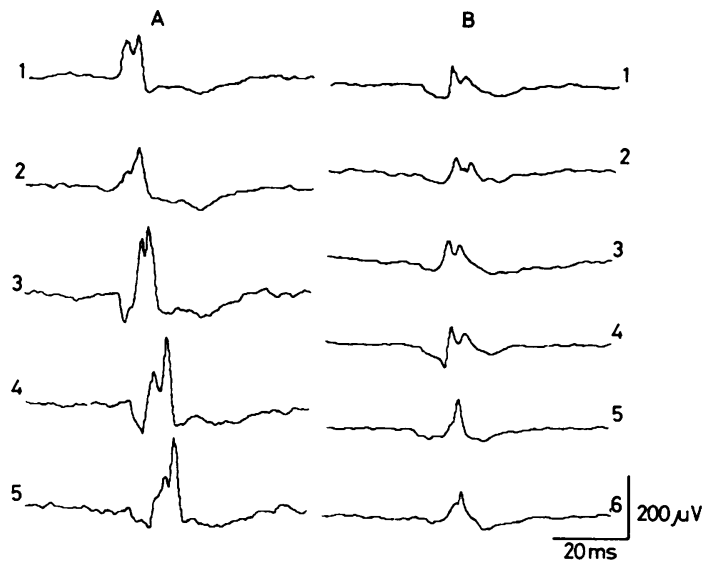

Fig 5 Two studies of multiple recording sites. (A) shows recordings from the same motor unit in $5 \mathrm{~mm}$ steps along its longitudinal axis, seen as an increasing latency from the trigger. The general shape remains. $(B)$ represents recordings from another motor unit when the electrode is moved perpendicularly to motor unit axis. The electrode was moved from 0 to $12 \mathrm{~mm}$ from the lateral border of the motor unit to the medial border. Trace 3 was taken at the end of the session as a control at position $5 \mathrm{~mm}$, corresponding to that of trace 2.

recording was used for triggering. The Macro electrode was positioned so that its small electrode recorded single muscle fibre action potentials which were time-locked to the triggering potentials. The Macro EMG recording was then performed. Keeping the same position for the triggering electrode the Macro electrode was repositioned in $5 \mathrm{~mm}$ steps along or across the long axis of the muscle corresponding to the region in the muscle used for routine studies (fig 5). The shape was essentially unchanged but could sometimes show definite variations between different positions. For the longitudinal recording sites the amplitude varied by $49.5 \pm$ $24.7 \%$ (range $21-106 \%, n=11$ ) (expressed as 100 maximum amplitude-minimum amplitude/mean (maximum amplitude + minimum amplitude)) between extreme values within one unit with the lowest values for electrode separation of $10 \mathrm{~mm}$ and the highest for the maximum electrode separation of $60 \mathrm{~mm}$. When the electrode was moved perpendicularly to the motor unit axis between the two most lateral positions, often about $15 \mathrm{~mm}$, at which single muscle fibre action potential from the same motor unit could be recorded the amplitude varied by $49 \cdot 2 \pm 18 \cdot 1 \%$ (range $23-72 \%, n=6)$.

\section{Discussion}

A number of recent studies have examined the changes of nerve, whole muscle and motor unit properties associated with age. ${ }^{6-9}$ The reduction in muscle bulk and strength in the elderly is reflected by a fall in amplitude of the supramaximally evoked muscle action potential ${ }^{710}$ and reduction in maximal isometric twitch tension developed by the whole muscle. ${ }^{7}$ However these effects become apparent mainly after the age of 60 there being little change over the earlier decades. The number of motor units as determined with the motor unit count method remains relatively stable in the small muscles of the hand and foot until the age of 60 . Thereafter there is a progressive decline in number of motor units with a concomittant increase in their size. ${ }^{78}$

Single Fibre EMG fibre density values which reflect the local anatomical organisation of the motor unit increase with age to differing degrees according to the muscle studied. ${ }^{4}$ In extensor digitorum brevis for example increases may be noted as early as the third decade ${ }^{11}$ while in tibialis anterior and extensor digitorum communis significant changes are not seen until after the ages of 50 and 60 respectively. In vastus lateralis and biceps brachii muscles slight changes are seen only after the age of 60 years.

The fibre density changes were accompanied by some increase in jitter and even blocking in a few of the recordings. The findings are interpreted as signs of slowly progressing reinnervation with remodelling of the motor unit.

The Macro EMG also changed with age in two of the three muscles examined. In both vastus lateralis and tibialis anterior there was a slight trend towards higher values particularly after the age of 60 .

What is the cause for the increase in the Macro MUP amplitude? The following are some comments about the factors determining the Macro EMG signal. The recording electrode probably gives rise to pronounced shunting effect of the electrical field of the closest muscle fibres. A fibrillation potential for example generates a "Macro MUP" of an amplitude of less than $5 \mu \mathrm{V}$ in comparison to the 100 to $300 \mu \mathrm{V}$ of the voluntarily activated motor unit. The electrode has a low impedance and consequently can rather faithfully record low frequency components enabling activity from more distant fibres to make significant contribution to the Macro signal. Furthermore the large electrode extends through many fascicles, traversing the whole motor unit, thereby increasing its chance of summating activity from the major part of the motor unit. The electrode therefore has a large uptake area, which probably includes the whole motor unit. This assumption is 
supported by the fact that the Macro MUP has often the same shape for different electrode positions within the motor unit. Certainly the most adjacent muscle fibre gives the largest contribution to the signal but simulation experiments show (Nandedkar, Stålberg, to be published) that by increasing the recording distances from 25 to $275 \mu \mathrm{m}$ the amplitude decreased to $54 \%$ for the Macro electrode, as compared to $14 \%$ for the SFEMG electrode.

The amplitude of the Macro MUP is determined by the average distance of the muscle fibres to the electrode, by the size of the muscle fibres and by the total number of muscle fibres within the motor unit, the volume characteristics of the muscle and the electrical properties of the recording system, including the electrode.

Because of the recording method the electrode is always within the territory of the motor unit and the average distance from all fibres varies by a factor of less than two from an optimal position in the middle of a motor unit to a position at the margin. The experiment where this was tested by inserting the electrode at different sites located perpendicularly to the main direction of the motor unit at the same level along the muscle fibres showed difference in amplitude values which agreed with this (about $50 \%$ difference between extreme amplitude values). Similar values are found in simulation experiments.

The second factor to be considered is fibre size, the significance of which we have seen in the lower than normal Macro MUP amplitudes recorded from muscles of patients with immobilisation atrophy compared to normal. With other parameters unchanged, a small muscle fibre will generate lower action potential at the electrode since its relative distance, measured in diameters, is increased.

A third factor is the number of muscle fibres. For the compound nerve action potential the number of generators seems to be more important than their individual size ${ }^{12}$ which may be true also in muscle.

The effect of the volume conduction properties of muscle tissue and of the electrode characteristics on the obtained signal is not yet known in detail, which makes it impossible to translate MUP amplitudes into exact numerical motor unit parameters. These factors vary probably less between subjects than other determinants of the recorded signal. The recording system is kept standardised and as for other types of electrodes, new normal values have to be determined.

What is the explanation for the large scatter of motor unit size observed? One is related to the localisation of the motor unit within the muscle. Separate studies showed a slight decrease in amplitudes with increasing recording depth in some subjects but no difference in others. In earlier versions of the Macro
EMG method the whole electrode was uninsulated and the amplitude reduction for deep motor units, more pronounced than with this electrode, was explained partly as an effect of increasing shunting effect when a larger part of the cannula was inserted in the muscle. With the present electrode the same amount of bare cannula was inside the muscle in all recordings and this effect is therefore eliminated. Different sizes of superficial and deep motor units, also indicated by other techniques seems to be the most likely explanation for the differences. ${ }^{13}$ In the present investigations efforts were made to obtain recordings equally representing all depths.

Another factor is the recruitment threshold. As known from other studies motor units recruited at a higher activation threshold tends to be larger. ${ }^{14}$ This is also seen in the Macro EMG since the mean amplitude may be of the order of five times for motor units recruited at $30 \%$ of maximum force as compared to those recruited below $10 \% .^{15}$

Wrich of the factors determining the amplitude of the Macro MUP may change with age? The uptake area and the effect of recording position in relation to the motor unit within the muscle should remain constant since the same recording principle is used for all ages. The change of muscle tissue properties is probably not an important factor since the increase with age is not a general shift towards higher amplitudes but usually the addition of Macro MUPs with high amplitudes (fig 2). Concerning differences in size with different thresholds, age changes may come into play since a loss of predominantly small motor units should lead to earlier activation of larger motor units resulting once more in a general shift towards higher amplitudes. As mentioned above this is not the case and small motor units are still represented within the sample.

Could a closer fibre packing due to changed muscle volume cause an increased Macro MUP since each fibre is now closer to the electrode? This factor should also cause a general increase in the amplitudes of all the motor units and cannot account for the considerable change in only some of the motor units.

The size of the Macro MUP is theoretically also dependent on the size of each muscle fibre. A general muscle fibre hypertrophy could therefore cause an increased amplitude. However evidence from histological studies in vastus lateralis in the same patients examined with Macro EMG showed no significant muscle fibre hypertrophy in the older subjects but rather a decrease in mean diameter after the age of 60 compared to lower ages (to be published).

Finally the amplitude also depends on the number of muscle fibres in the motor unit. In the case of 
reinnervation the number of muscle fibres increases in the remaining motor units. Evidence from our own biopsy studies showing fibre type grouping in vastus lateralis (to be published) and an increase in fibre density indicates at least a local change in the motor units similar to that seen after known neurogenic lesions. These findings suggest that reinnervation is the most likely mechanism for the increase in Macro MUP amplitude. Furthermore the increase in amplitude implies that the surviving motor units actually increase in size due to an overall increase in the number of fibres, and that there is not just reorganisation of the motor unit with local drop out of fibres and local reinnervation. This increase in size is obviously a very effective compensatory mechanism and can postpone the development of clinical weakness and muscle wasting.

One further aspect to emerge from the study is the slight and, below the age of 60 , significant difference of motor unit size between men and women. It is a common observation that muscle strength is greater in men than women, and the present findings suggest that this may partly be due to larger motor units in men. Whether this is mainly due to more fibres or larger fibres is under study. In the elderly where the difference is more pronounced, the changes in motor unit size secondary to nerve trauma may be more marked in men than women due to different occupational demands.

The evidence from Scanning EMG ${ }^{15}$ suggests that the peaks seen on Macro EMG correspond to fractions of the motor unit each of which may have a localised collection of end-plates probably innervated by a major nerve branch. The time interval between the peaks describes the dispersion in time of the fronts of the electrical activity in the motor unit fractions. An increased time between peaks or dispersion of the Macro signal as a whole may be caused by the fact that the recording electrode is not parallel to the end-plate zone. This possibility can be examined in the scanning EMG recordings. A nonparallel situation should give a continous shift of the latency of the action potentials within the motor unit. This was tested with the standard recording position used for the Macro electrode. The time between the peaks was not due to a systematic change in latency since they occurred randomly within the motor unit. The dispersion of the Macro MUP is due to the varying transmission time along the nerve branches, the scatter of the motor endplates in the motor unit and the difference in propagation velocity for individual muscle fibres. No major changes in duration are likely to be found with age since the amplitude and area change in parallel with age in this material. The number of peaks and the time between the peaks does not increase with age. This indicates that reinnervation does not result from the formation of new major nerve branches since this should probably be seen as new motor unit fractions, but rather that reinnervation occurs by terminal axonal sprouting and therefore does not cause much increase in total Macro MUP duration.

The reasons for the differences in degree of age changes noted in the muscles studied are unknown. Factors such as ventral horn cell degeneration, peripheral nerve degeneration, entrapment at root or more distal sites and repeated nerve traumata have to be considered. The combination of these factors vary for different muscles.

In summary the Macro EMG motor unit potentials increase with age more in distal than in proximal muscles. The changes with age are most likely due to reinnervation after a preceding denervation. The changes are of such a magnitude that the age has to be taken into account when the method is used to study motor units in different neuromuscular disorders.

This work was supported by the Swedish Medical Research Council, Grant No 135 (ES) and by the Medical Research Council (UK), Travelling Fellowship (PRWF). Eng Lars Antoni made the computer programs and Eng Gösta Loven the Macro EMG electrode. Annika Alsterlind and Kerstin Danielsson lent skilful assistance.

\section{References}

${ }^{1}$ Thiele B, Bohle A. Anzahl der Spike-Komponenten im Motor-Unit Pontential. EEG EMG 1978;9:125-30.

${ }^{2}$ Stålberg E. Macro EMG, a new recording technique. $J$ Neurol Neurosurg Psychiatry 1980;43:475-82.

${ }^{3}$ Stålberg E, Antoni L. Microprocessors in the analysis of the motor unit and neuromuscular transmission. In: Yamaguchi N, Fujisawa K, eds. Recent Advances in EEG and EMG Data Processing. Amsterdam: Elsever/North-Holland Biomedical Press. 1981; 295-313.

${ }^{4}$ Stålberg E, Trontelj J. Single Fibre Electromyography. Woking, England: Mirvalle Press, 1979.

${ }^{5}$ Nandedkar S, Kim Y, Sanders D, Anne A. Signal representation of Macro EMG. IEEE/Engineering in Medicine and Biology Society 2nd Annual Conference 1980; 296-300.

' Burke WE, Tuttle WW, Thompson CW, Janney CD, Weber RJ. The relation of grip strength and gripstrength endurance to age. J Appl Physiol 1953;5:628-30.

${ }^{7}$ Cambell MJ, McComas AJ, Petito F. Physiological changes in age muscles. $J$ Neurol Neurosurg Psychiatry 1973;36:174-82.

${ }^{8}$ Brown WF. Functional compensation of human motor units in health and disease. $J$ Neurol Sci 1973;20:199-209. 
9 Jennekens FGI, Tomlinson BE, Walton JN. Histochemical aspects of five limb muscles in old age. An anatopsy study. J Neurol Sci 1971;14:259-76.

${ }^{10}$ Ludin H-P. Praktische Electromyographie. Stuttgart: Ferdinand Enke. 1976; 131-60.

$"$ Stâlberg E, Thiele B. Motor unit fibre density in the extensor digitorum communis muscle. J Neurol Neurosurg Psychiatry 1975;38:874-80.

${ }^{12}$ Lambert EH, Dyck PJ. Compound action potentials of sural nerve in vitro in peripheral neuropathy. In: Dyck PJ, Thomas PK, Lambert EH, eds. Peripheral
Neuropathy. Philadelphia: Saunders, 1975;1:427-41.

${ }^{13}$ Clamann HP. Activity of single motor units during isometric tension. Neurology (Minneap) 1970;20:254-60.

${ }^{14}$ Milner Brown HS, Stein RB, Yemm R. The orderly recruitment of human motor units during voluntary contractions. J Physiol (Lond) 1973;230:359-70.

${ }^{15}$ Stålberg E, Antoni L. Electrophysiological cross section of the motor unit. J Neurol Neurosurg Psychiatry 1980;43:469-74. 